\title{
New drugs for an ancient parasite
}

\author{
Alex Loukas \& Jeffrey M Bethony
}

Only one drug is widely used to treat schistosomiasis, a chronic, neglected tropical disease caused by parasitic blood flukes. Fears of potential drug resistance have accelerated the search for new classes of antischistosome drugs. A promising candidate has now emerged (pages 407-412).

Schistosomiasis, caused by blood flukes of the genus Schistosoma (Fig. 1), has afflicted humans for thousands of years and is arguably the most important human helminth infection. Schistosomes infect more than 200 million people in 76 countries and are responsible for up to 20,000 deaths per year.

Eggs of the parasite become trapped in tissues such as the bowel or bladder, leading to chronic immunopathology.

Recent assessments of the public health impact of schistosomiasis include not only gross organ pathology, but also the anemia, pain, diarrhea, exercise intolerance and undernutrition that results from chronic infection, raising the estimate of disability previously assigned to the disease by the World Health Organization (WHO) $)^{1,2}$. Schistosomiasis can be treated with anthelmintic agents, but the current reliance on a single drug, praziquantel, has prompted fears of the emergence of drug-resistant parasites.

In this issue of Nature Medicine, Sayed et al. ${ }^{3}$ provide hope for the development of new antischistosome drugs. By targeting the antioxidant pathway, the authors identified a family of compounds that interrupt the ability of the parasite to detoxify reactive oxygen species and kill schistosomes in vitro and in animal models of infection. If their results hold true in human clinical trials, this class of compound has the potential to become a

Alex Loukas is in the Division of Infectious Diseases, Queensland Institute of Medical Research, 300 Herston Road, Brisbane, QLD 4006, Australia. Jeffrey M. Bethony is in the Department of Microbiology, Immunology and Tropical Medicine, George Washington University, 2300 Eye Street Northwest, Washington, DC 20037, USA. e-mail: Alex.Loukas@qimr.edu.au

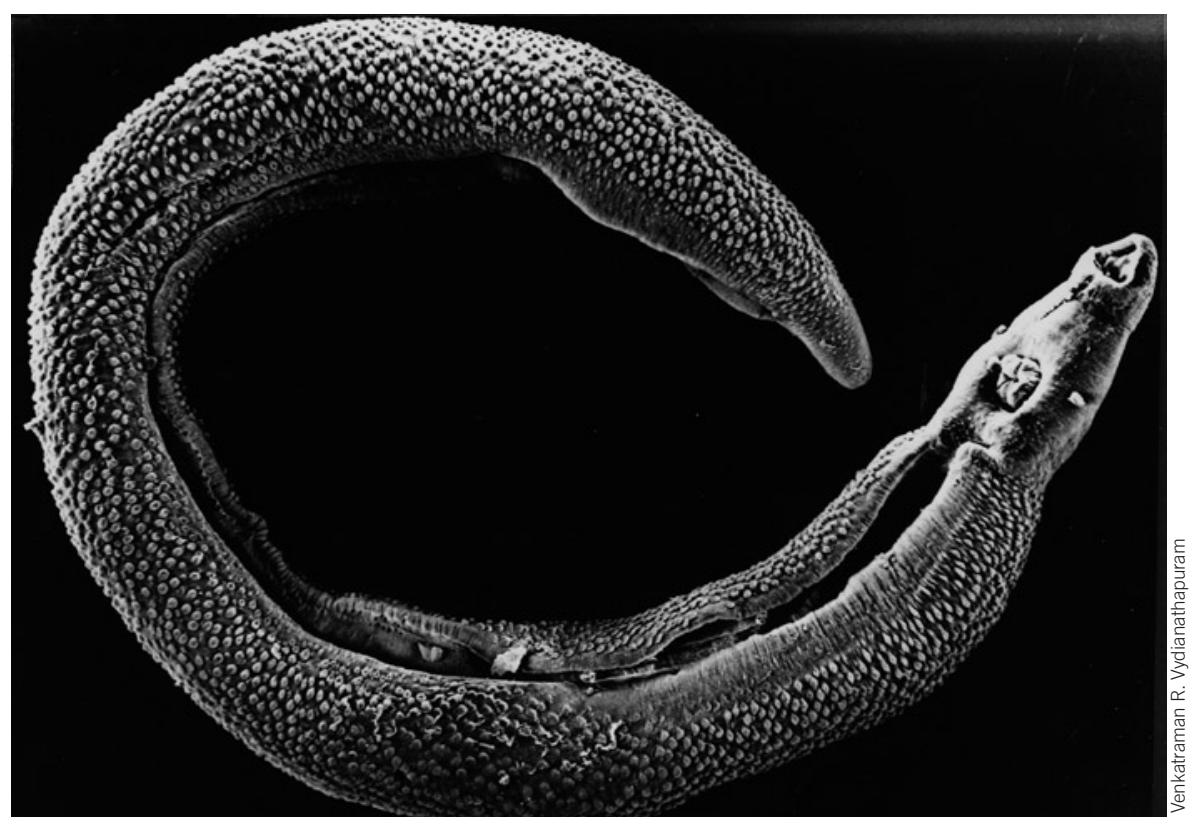

Figure 1 An adult male Schistosoma mansoni.

powerful new tool for the control of schistosomiasis.

The mainstay of current schistosomiasis control is the anthelmintic drug praziquantel (PZQ), a quinoline compound developed in the 1970s by Bayer as an anthelmintic drug for veterinary parasites. PZQ causes calcium ion fluxes, which results in contraction of parasite muscle followed by paralysis and death of the worm. The WHO places PZQ on its 'Essential Medicines' register, which catalogs "minimum medicine needs for

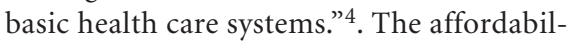
ity, efficacy and safety of PZQ prompted the formation of the Schistosomiasis Control Initiative, an organization funded by the Bill and Melinda Gates Foundation that currently facilitates mass administration of PZQ in six African countries. Some people in the Schistosomiasis Control Initiative believe that mass administration of PZQ will consign schistosomiasis to history by $2015^{5}$, but concerns about the sustainability of these efforts remain. This is because of lower-than-expected efficacies of singledose PZQ, high rates of reinfection after treatment and the potential emergence of anthelminthic drug resistance. Indeed, PZQ resistance has already been documented in the laboratory ${ }^{6}$. Given such weaknesses in this single-drug regimen, there is a pressing need for new control tools.

Alternative drugs are available to treat schistosomiasis, but they have drawbacks. 


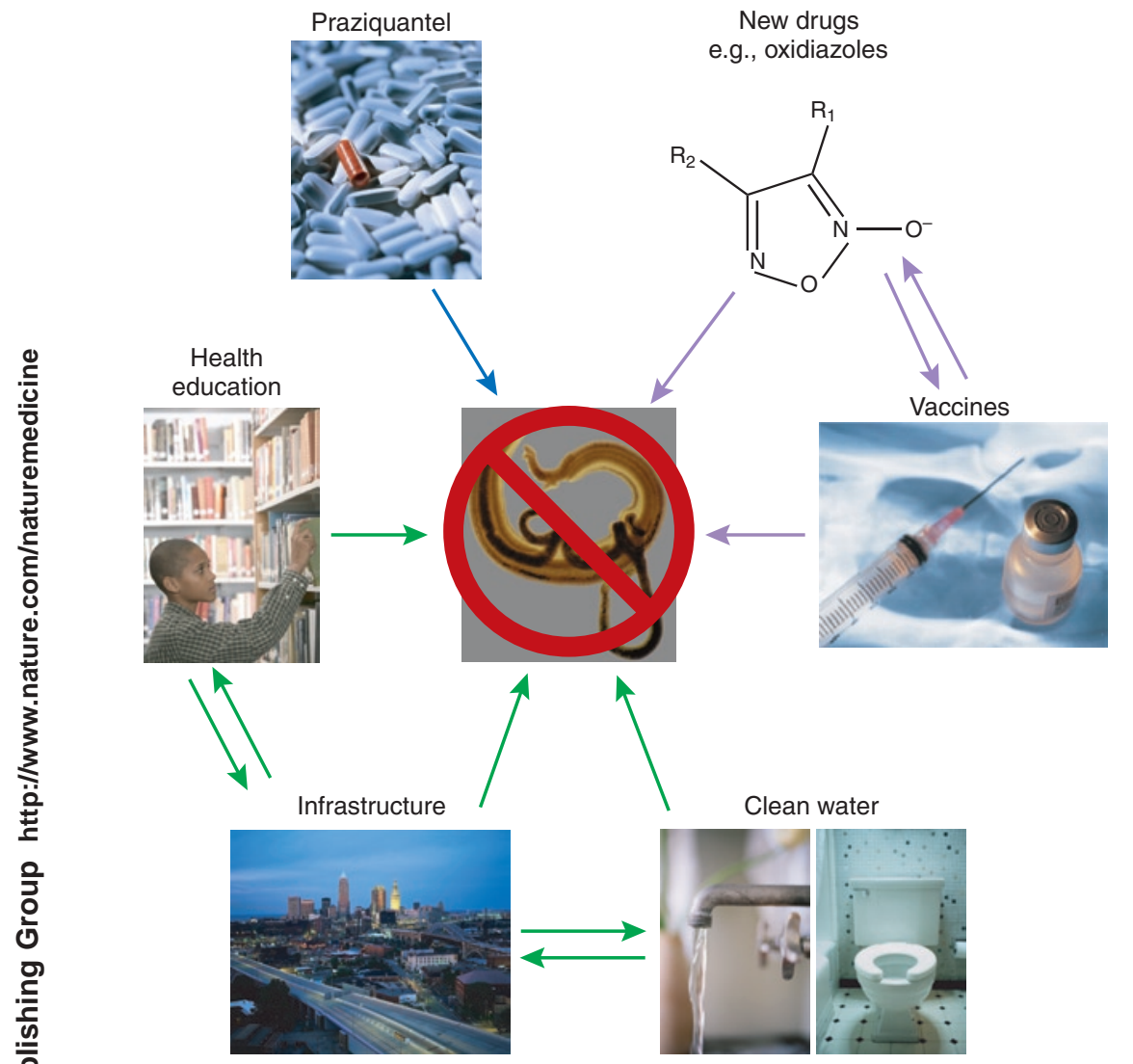

Figure 2 An integrated approach is needed to eliminate schistosomiasis. Linking vaccination with chemotherapy, together with a program of clean water and health education, would offer the most effective long-term approach to reducing infection and morbidity from schistosomiasis. This requires short-term goals (purple arrows) and long-term, often more ambitious, goals (green arrows).

Artemether (artemisinin) is derived from a Chinese plant and can treat a range of parasitic infections. Although artemether is safe and effective against schistosomes, a major obstacle to its use is that it is considered the last line of defense for treating multidrugresistant malaria, which is often coendemic with schistosomiasis, especially in Africa, and thus using it to treat schistomsomiasis may result in artemether-resistant malaria.

The present discovery of the new compounds is the result of the first assay officially accepted for screening by the US National Institutes of Health Molecular Libraries Initiative, a partnership developed to enable academic researchers to apply a biotech company-type approach to drug discovery and preclinical testing. The new compounds target an enzyme involved in detoxifying antioxidants in the parasites.

Schistosomes are unique in the way they deal with antioxidants, both their own and those generated by the host's immune system. Vertebrates detoxify reactive oxygen species using two distinct enzymes-glutathione reductase and thioredoxin reductase. Sayed et al. ${ }^{3}$ built upon their previous biochemical and bioinformatic data, which supported the notion that $S$. mansoni uses just one unique, multifunctional detoxifying enzyme. This enzyme, thioredoxin-glutathione reductase (TGR), has both glutathione reductase and thioredoxin reductase activities ${ }^{8}$. Using a fully automated, high-throughput format, the authors screened 71,028 compounds for TGR inhibitory activity ${ }^{9}$. Several compounds that were active in the low micromolar to nanomolar ranges were identified, including the oxadiazole 2 -oxides 9 .

Sayed et al. ${ }^{3}$ showed that the oxadiazole 2-oxide 4-phenyl-1,2,5-oxadiazole-3-carbonitrile-2-oxide (which they refer to as compound 9) irreversibly bound to TGR and inhibited its enzymatic activity, probably via modification of cysteine or selenocysteine residues in the protein. Most importantly, these new oxadiazoles (particularly compound 9) held their own against PZQ and artemether: in vitro they could kill all intramammalian developmental stages of schistosomes (skin and lung larvae as well as juvenile and adult worms). In contrast, PZQ and artemether target mainly the adult and larval stages, respectively.
Compound 9 was tested for efficacy in a mouse model of $S$. mansoni infection, and $88-99 \%$ reductions in worm burdens were obtained irrespective of the timing of the treatment. Moreover, the activity of compound 9 surpassed all criteria set forth by the WHO for potential new drugs for schistosomiasis. Compound 9 was also equally efficacious against two other common human schistosomes, Schistosoma haematobium and Schistosoma japonicum. S. haematobium is the cause of urogenital schistosomiasis (which can result in bladder cancer), and this parasite is responsible for the majority of schistosome-related morbidity in Africa ${ }^{10}$. Although less prevalent than the African species, S. japonicum is an aggressive parasite in terms of pathology; however, the burden of disease associated with it throughout Asia is not well established $^{2}$.

A collaboration between academic laboratories developed and funded this research, so it seems that similar partnerships could ensure the progression of this drug through the development pipeline and into clinical trials. Public-private partnerships are proving to be a successful model for the development of new drugs for neglected tropical diseases ${ }^{11}$. Such organizations are crucial, given the lack of interest by large pharmaceutical companies in developing medicines without a large commercial market.

It is, however, important to emphasize that drugs are only one link in the chain of sustainable schistosomiasis control (Fig. 2). Emphasis should be placed on integrated mechanisms that prevent, as well as cure, schistosomiasis to achieve eradication.

Vaccines are an essential component in such a sustainable control program and offer the ability to prevent infection and reinfection, especially when coadministered with anthelmintic drugs. Indeed, recent advances in genomics and proteomics have resulted in the discovery of new antigens that are showing promise as recombinant vaccines and are in various stages of clinical development ${ }^{12}$. Moreover, a truly integrated approach to control would include infrastructure and education components. Such an integrated approach would require longer-term investments, but the consequences would reach far beyond the control of just schistosomiasis.

Despite a dearth of funding for basic schistosomiasis research from major philanthropic organizations, it is reassuring to see that groundbreaking advances are still being made to control this illness-one of the most crippling and unrelenting of the tropical diseases. 
1. King, C.H., Dickman, K. \& Tisch, D.J. Lancet 365 1561-1569 (2005).

2. Finkelstein, J.L., Schleinitz, M.D., Carabin, H. \& McGarvey, S.T. PLoS Negl. Trop. Dis. 2, e158 (2008).

3. Sayed, A.A. et al. Nat. Med. 14, 407-412 (2008).

4. World Health Organization. WHO Model List of Essential Medicines, 15th edn., 1-27 (World Health
Organization, Geneva, 2007).

5. Fenwick, A. Science 313, 1077-1081 (2006).

6. Fallon, P.G. \& Doenhoff, M.J. Am. J. Trop. Med. Hyg. 51, 83-88 (1994).

7. Inglese, J. et al. Proc. Natl. Acad. Sci. USA 103, 11473-11478 (2006).

8. Alger, H.M. \& Williams, D.L. Mol. Biochem. Parasitol. $121,129-139$ (2002).
9. Simeonov, A. et al. PLoS Negl. Trop. Dis. 2, e127 (2008)

10. van der Werf, M.J. et al. Acta Trop. 86, 125-139 (2003).

11. Croft, S.L. Trans. R. Soc. Trop. Med. Hyg. 99 Suppl 1, S9-S14 (2005).

12. McManus, D.P. \& Loukas, A. Clin. Microbiol. Rev. 21 225-242 (2008).

\title{
Dropping acid to help cystic fibrosis
}

\author{
Gerald B Pier
}

\section{Vesicular accumulation of the membrane component ceramide may underlie key aspects of lung pathology in cystic fibrosis (pages 382-391).}

Breathing is basic-as anyone who has ever struggled for adequate oxygen, which is virtually everyone, can readily attest. Compromises to the lung that affect oxygenation of tissues can seriously threaten health. The lungs, like other mucosal surfaces, are major ports of entry for microbes, but mammals must keep their lungs sterile, unlike the gastrointestinal or vaginal mucosa. They do so by relying on an effective innate and adaptive immune system to rapidly clear pathogens while minimizing consequences from inflammatory responses.

The hereditary disease cystic fibrosis occurs as a consequence of having two defective alleles of the gene encoding an epithelial chloride channel, the cystic fibrosis transmembrane conductance regulator (CFTR). Both chronic infection and chronic inflammation collude to destroy lung tissues, reduce the ability of affected people to breathe adequately and bring about an early death, often before 40 years of age, primarily owing to respiratory failure. How mutations in CFTR actually cause lung disease on a molecular and cellular level is still an area of intense investigation, particularly with regard to whether an absence of functional CFTR alone causes inflammation in the lung and to why one single pathogen, the Gramnegative bacterium Pseudomonas aeruginosa, is such an overwhelming cause of destructive infection and inflammation.

Findings in this issue of Nature Medicine address a key aspect of these questions. Teichgräber et al. ${ }^{1}$ find that the membrane component ceramide accumulates abnor-

Gerald B. Pier is at Brigham and Women's Hospital, Harvard Medical School, 181 Longwood Ave, Boston, Massachusetts 02115, USA. e-mail: gpier@rics.bwh.harvard.edu

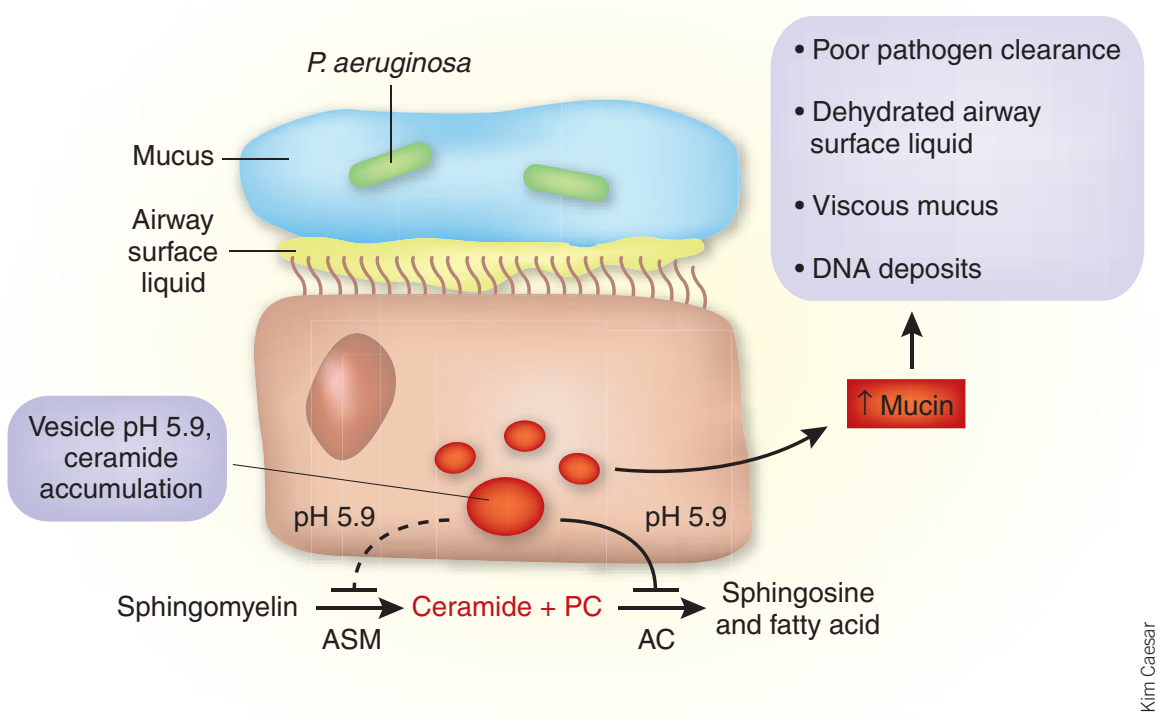

Figure 1 Enhancing inflammation and infection in the cystic fibrosis-affected airway. Synthesis of ceramide from sphingomyelin by acid sphingomyelinase (ASM) and degradation by acid ceramidase (AC) is optimal at an acidic $\mathrm{pH}$, such as that of normal intracellular vesicles ( $\mathrm{pH} 4.5)$. Lack of functional CFTR increases vesicle $\mathrm{pH}$ to 5.9, partially inhibiting ASM and highly inhibiting AC, resulting in ceramide accumulation in the vesicle and age-dependent pulmonary inflammation. Other properties of the cystic fibrosis-affected lung, including lack of the CFTR receptor needed for clearing Pseudomonas aeruginosa, enhanced mucin secretion, a dehydrated airway surface liquid, viscous mucus and DNA deposits released from ceramide-engorged apoptotic cells, also contribute to $P$. aeruginosa airway colonization. Eventually, this microbe establishes a chronic infection wherein bacterial cells reside in low-oxygen mucus plugs and become highly resistant to clearance or killing by host defenses.

mally in respiratory epithelial cells of transgenic cystic fibrosis mice and is also present in large amounts in nasal epithelial cells and lung tissues from humans with cystic fibrosis. Ceramide seems to induce endogenous inflammation while also enhancing the susceptibility of older cystic fibrosis mice to lung infection with $P$. aeruginosa. Ceramide accumulation contributes to the pathology of the cystic fibrosis lung by promoting inflammatory cytokine production, cellular apoptosis and DNA accumulation, which serve as a nidus for initiating $P$. aeruginosa infection.

The authors sought to address an outstanding question in cystic fibrosis research, which is whether the lack of functional CFTR induces an inflammatory state in the lung, even in the absence of infection ${ }^{1}$. Teichgräber et al. ${ }^{1}$ focused on sphingolipids, as they are known regulate the production of molecules that cause lung inflammation and affect cell 